\title{
Hardness Results for Homology Localization
}

\author{
Chao Chen · Daniel Freedman
}

Received: 17 June 2009 / Revised: 29 October 2010 / Accepted: 17 December 2010 /

Published online: 14 January 2011

(C) Springer Science+Business Media, LLC 2011

\begin{abstract}
We address the problem of localizing homology classes, namely, finding the cycle representing a given class with the most concise geometric measure. We study the problem with different measures: volume, diameter and radius.

For volume, that is, the 1-norm of a cycle, two main results are presented. First, we prove that the problem is NP-hard to approximate within any constant factor. Second, we prove that for homology of dimension two or higher, the problem is NP-hard to approximate even when the Betti number is $O(1)$. The latter result leads to the inapproximability of the problem of computing the nonbounding cycle with the smallest volume and computing cycles representing a homology basis with the minimal total volume.

As for the other two measures defined by pairwise geodesic distance, diameter and radius, we show that the localization problem is NP-hard for diameter but is polynomial for radius.

Our work is restricted to homology over the $\mathbb{Z}_{2}$ field. Results over other fields have been studied recently by Dey et al.: In STOC, pp. 221-230 (2010).
\end{abstract}

Partially supported by the Austrian Science Fund under grant P20134-N13.

C. Chen $(\otimes)$

Institute of Science and Technology, Vienna, Austria

e-mail: chao.chen@ist.ac.at

C. Chen

Vienna University of Technology, Vienna, Austria

D. Freedman

School of Engineering, Bar Ilan University, Ramat Gan, Israel

e-mail: d.e.freedman@gmail.com 


\section{Introduction}

The problem of computing the topological features of a space has recently drawn much attention from researchers in various fields, such as high-dimensional data analysis [5], graphics [17], networks [12] and computational biology [11]. Topological features are often preferable to purely geometric features, as they are more qualitative and global, and tend to be more robust. If the goal is to characterize a space, therefore, features which incorporate topology seem to be good candidates.

While topological features are global, the need to "localize" them has been raised in a variety of applications. In graphics and manifold learning, one wants to detect and remove topological noise such as the small holes and handles that are introduced in data acquisition; this is often done in the context of traditional signal-noise analysis and finite sampling of continuous spaces [21, 25, 29]. In the area of sensor networks, holes of the coverage region, caused by physical constraints, should be accurately identified and described so as to produce a network as robust as possible [20, 26]. In the study of shape, 3D shapes may be enriched with properties such as curvatures associated with tangent vectors at each tangent plane. The new augmented shape lives in high dimension, whose topological features can be localized and reveal geometric features of the original shape [4].

In this paper, we will address the localization problem, namely, finding the smallest representative cycle of a homology class with regard to a given natural criterion of the size of a cycle. The criterion should be deliberately chosen so that the corresponding smallest cycle is concise in not only mathematics but also intuition. Such a cycle is a "well-localized" representative cycle of its class. See Fig. 1 for examples. In a disk with three holes (Fig. 1(a)), cycles $z_{1}$ and $z_{2}$ are well localized; $z_{3}$ is not. In a 2-handled torus (Fig. 1(b)), the concise cycle $z_{1}$ is a better representative (than $z_{2}$ ) of its class and describes the small handle better.

In Sect. 4.1, we use volume, the number of simplices of a cycle, as the criterion to minimize. For a one-dimensional (resp. two-dimensional) cycle, the volume is its length (resp. area).

We have two main results. First, we prove that localizing a given class with the minimal volume cycle is NP-hard to approximate within any constant factor. The

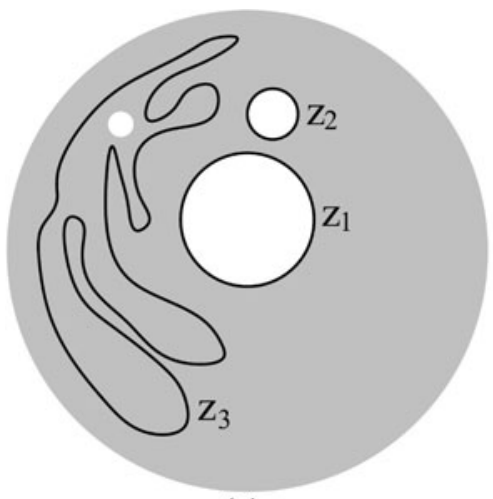

(a)

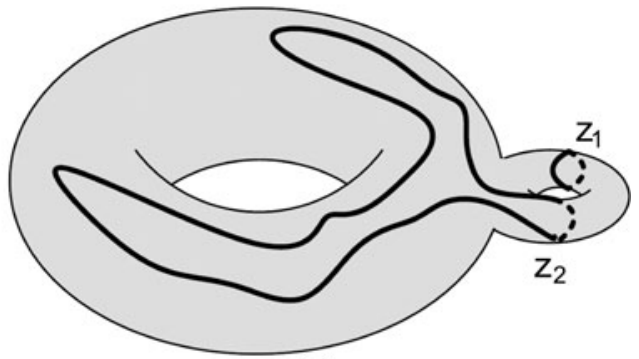

(b)

Fig. 1 Motivating examples for localization 
proof is a strict reduction from the nearest codeword problem [2]. We prove the inapproximability for homology of any dimension.

Second, we prove that for homology of dimension two or higher, computing the nonbounding cycle with the smallest volume is NP-hard to approximate within any constant factor. This is true even when the Betti number is fixed. This result leads to the inapproximability of two other problems concerning homology of dimension two or higher, namely,

- localizing a given class with the minimal volume cycle, when Betti number is fixed, and

- computing a homology cycle basis with the minimal total volume.

We conclude the paper with a discussion of other minimization criteria, including diameter and radius (Sect. 5). In specific, we show that localization with diameter cost function is NP-hard, where as with radius is polynomial.

Throughout this paper, the topological features we use are homology classes over $\mathbb{Z}_{2}$ field. (Thus, all the additions are mod 2 additions.)

\section{Preliminaries}

\subsection{Homology Groups}

We briefly describe some background knowledge from algebraic topology. Refer to [24] for more details. We restrict our discussion to the combinatorial framework of simplicial homology over $\mathbb{Z}_{2}$ field.

Given a simplicial complex $K$, a $d$-chain is a formal sum of $d$-simplices, $c=$ $\sum_{\sigma \in K} a_{\sigma} \sigma, a_{\sigma} \in \mathbb{Z}_{2}$. All the $d$-chains form the group of $d$-chains, $\mathrm{C}_{d}(K)$. The boundary of a $d$-chain is the sum of the $(d-1)$-faces of all the $d$-simplices in the chain. The boundary operator $\partial_{d}: \mathrm{C}_{d}(K) \rightarrow \mathrm{C}_{d-1}(K)$ is a group homomorphism.

A $d$-cycle is a $d$-chain without boundary. ${ }^{1}$ The set of $d$-cycles forms a subgroup of the chain group, which is the kernel of the boundary operator, $Z_{d}(K)=\operatorname{ker}\left(\partial_{d}\right)$. A $d$-boundary is the boundary of a $(d+1)$-chain. The set of $d$-boundaries forms a group, which is the image of the boundary operator, $\mathrm{B}_{d}(K)=\operatorname{img}\left(\partial_{d+1}\right)$. It is not hard to see that a $d$-boundary is also a $d$-cycle. Therefore, $\mathrm{B}_{d}(K)$ is a subgroup of $\mathrm{Z}_{d}(K)$. A $d$-cycle which is not a $d$-boundary, $z \in \mathrm{Z}_{d}(K) \backslash \mathrm{B}_{d}(K)$, is a nonbounding cycle. In our case, the coefficients belong to a field, namely $\mathbb{Z}_{2}$; when this is the case, the groups of chains, boundaries and cycles are all vector spaces. Note that this is not true when the homology is over a ring which is not a field, such as $\mathbb{Z}$.

The $d$-dimensional homology group is defined as the quotient group $\mathrm{H}_{d}(K)=$ $\mathrm{Z}_{d}(K) / \mathrm{B}_{d}(K)$. An element in $\mathrm{H}_{d}(K)$ is a homology class, which is a coset of $\mathrm{B}_{d}(K)$, $[z]=z+\mathrm{B}_{d}(K)$ for some $d$-cycle $z \in \mathrm{Z}_{d}(K)$. If $z$ is a $d$-boundary, $[z]=\mathrm{B}_{d}(K)$ is the identity element of $\mathrm{H}_{d}(K)$. Otherwise, when $z$ is a nonbounding cycle, $[z]$ is a nontrivial homology class, and $z$ is called a representative cycle of [z]. Cycles in the

\footnotetext{
${ }^{1}$ For those unfamiliar with homology, we emphasize that a 1-cycle is different from the cycle defined in graph theory. For the former definition, a 1-cycle can be a disjoint union of arbitrarily many 1-cycles. But this is not true for the latter definition.
} 
same homology class are homologous to each other, which means their difference is a boundary.

The dimension of the homology group, which is referred to as the Betti number,

$$
\beta_{d}=\operatorname{dim}\left(\mathrm{H}_{d}(K)\right)=\operatorname{dim}\left(\mathrm{Z}_{d}(K)\right)-\operatorname{dim}\left(\mathrm{B}_{d}(K)\right) .
$$

As the dimension of the chain group is upperbounded by the cardinality of $K, n$, so are the dimensions of $\mathrm{B}_{d}(K), \mathrm{Z}_{d}(K)$ and $\mathrm{H}_{d}(K)$. The Betti number can be computed with a reduction algorithm based on row and column operations of the boundary matrices [24]. Various reduction algorithms have been devised for different purposes.

A homology basis is a set of $\beta_{d}$ classes generating the group $\mathrm{H}_{d}(K)$. We call a set of $\beta_{d}$ nonbounding cycles representing a homology basis a homology cycle basis. Any $d$-cycle can be written as a linear combination of a homology cycle basis and boundaries. Given a homology cycle basis $\left\{z_{1}, z_{2}, \ldots, z_{\beta_{d}}\right\}$, any homology class can be written as $\sum_{i=1}^{\beta_{d}} a_{i}\left[z_{i}\right]$. Its cycles can be written as $\left[z_{1}, \ldots, z_{\beta_{d}}\right] \alpha+\partial_{d+1} \gamma$, where $\alpha=\left[\begin{array}{c}a_{1} \\ \vdots \\ a_{\beta_{d}}\end{array}\right]$.

Note that since the field is $\mathbb{Z}_{2}$, the set of $d$-chains is in one-to-one correspondence with the set of subsets of the set of $d$-simplices. A $d$-chain corresponds to an $n_{d^{-}}$ dimensional vector, whose nonzero entries correspond to the included $d$-simplices. Here $n_{d}$ is the number of $d$-simplices in $K$. Computing the boundary of a $d$-chain corresponds to multiplying the chain vector with a boundary matrix $\left[b_{1}, \ldots, b_{n_{d}}\right]$, whose column vectors are boundaries of $d$-simplices in $K$. By slightly abusing notation, we call the boundary matrix $\partial_{d}$.

We call a subset of simplices of a given simplicial complex a subcomplex if this subset itself is a simplicial complex. We define the $d$-skeleton of $K$ as the subcomplex consisting of all the $d$-simplices and their faces. The following notation will prove convenient. We say that a $d$-chain $c \in \mathrm{C}_{d}(K)$ is carried by a subcomplex $K_{0}$ when all the $d$-simplices of $c$ belong to $K_{0}$. We denote by vert $(K)$ the set of vertices of the simplicial complex $K$ and by vert $(c)$ that of the chain $c$. Denote by $|K|$ the underlying space of $K$ and by $|c|$ that of the chain $c$.

Replacing simplices by their continuous images in a given topological space gives singular homology. The simplicial homology of a simplicial complex is naturally isomorphic to the singular homology of its geometric realization. This implies, in particular, that the simplicial homology of a space does not depend on the particular simplicial complex chosen for the space. In figures of this paper, we often ignore the simplicial complex and only show the continuous images of chains.

The Discrete Geodesic Distance To introduce the diameter and radius functions, we need a notion of distance. As we will deal with a simplicial complex $K$, it is most natural to introduce a discrete metric and corresponding distance functions. We define the discrete geodesic distance from a vertex $p \in \operatorname{vert}(K), f_{p}$ : $\operatorname{vert}(K) \rightarrow \mathbb{R}$, as follows. Suppose that each edge in $K$ has a nonnegative weight and, for any vertex $q \in \operatorname{vert}(K), f_{p}(q)=\operatorname{dist}(p, q)$ is the length of the shortest path connecting $p$ and $q$ in the 1-skeleton of $K$. We may then extend this distance function from vertices to higher-dimensional simplices naturally. For any simplex $\sigma \in K$, 
$f_{p}(\sigma)$ is the maximal function value of the vertices of $\sigma, f_{p}(\sigma)=\max _{q \in \operatorname{vert}(\sigma)} f_{p}(q)$. This extension has the same effect as linearly interpolating the function on the interiors of the simplices (the sublevel sets of the two extensions are homotopy equivalent). Finally, we define the geodesic ball $B_{p}^{r}, p \in \operatorname{vert}(K), r \geq 0$, as the subset of $K$, $B_{p}^{r}=\left\{\sigma \in K \mid f_{p}(\sigma) \leq r\right\}$. It is straightforward to show that these subsets are in fact subcomplexes.

\subsection{Terminology from Coding Theory}

We focus on binary linear codes and thus only use matrices over the $\mathbb{Z}_{2}$ field. For consistency, we switch the roles of the row and column indices from the standard definition. Refer to [23] for details.

Given an $m \times k(m>k)$ full-rank matrix $A$, we define a linear code as the $k$ dimensional column space of $A$, namely, $\operatorname{span}(A)$. Each element of the linear code is called a codeword. This matrix is called the generator matrix as it is a basis of the linear code. By slightly abusing notation, we call a full-rank matrix $A^{\perp}$ the paritycheck matrix if its nullspace is the linear code generated by $A$.

Given a generator matrix $A, A^{\perp}$ may be computed in polynomial time. Its dimension is $(m-k) \times m$. In specific, $A^{\perp} A=0$, and thus $A^{T}\left(A^{\perp}\right)^{T}=0$. Any basis of the solution space of the linear equation system $A^{T} x=0$ would form a valid $A^{\perp}$. This could be computed by a Gauss-Jordan elimination. For example, the generator matrix and its parity-check matrix could be

$$
A=\left[\begin{array}{lll}
1 & 1 & 0 \\
0 & 1 & 0 \\
1 & 0 & 1 \\
1 & 1 & 0 \\
1 & 0 & 0
\end{array}\right], \quad A^{\perp}=\left[\begin{array}{ll}
1 & 1 \\
0 & 1 \\
0 & 0 \\
1 & 0 \\
0 & 1
\end{array}\right] .
$$

\subsection{The Hardness of Approximability and Strict Reductions}

We will prove that several optimization problems are NP-hard to approximate within any constant factor. Relevant definitions will be presented in this section. See [3] for more details. For ease of exposition, we only discuss minimization problems. The definitions can be extended to maximization problems easily.

An NP optimization problem $\Pi$ is a three-tuple $(\mathcal{I}$, Sol, $m)$ in which $\mathcal{I}$ is the set of instances. For each instance $I \in \mathcal{I}$, $\operatorname{Sol}(I)$ denotes the set of feasible solutions of $I$, and the cost function $m(I, S)$ produces a value for each feasible solution $S \in \operatorname{Sol}(I)$. Any instance can be recognized in time polynomial in its size, $\operatorname{card}(I)$. It is also polynomial to verify whether any given $S$ is a feasible solution or evaluate the cost function $m$.

For an instance $I$ and one of its feasible solutions $S \in \operatorname{Sol}(I)$, we define the performance ratio $\rho_{\Pi}(I, S)$ as the ratio of the value $m(I, S)$ (assume that $m(\cdot, \cdot) \geq 0$ ) over the value of the optimal solution; formally,

$$
\rho_{\Pi}(I, S)=\frac{m(I, S)}{m\left(I, S^{*}(I)\right)},
$$


where $S^{*}(I)$ is the optimal solution of $I$. The quality of a polynomial approximation algorithm $A$ is measured by the approximation ratio $\rho_{A}(I)=\rho_{\Pi}(I, A(I))$. For minimization problems, therefore, the approximation ratio is in $[1, \infty)$.

An NP optimization problem $\Pi$ belongs to the class APX if there exists a polynomial approximation algorithm $A$ and a value $r \in \mathbb{Q}$ such that given any instance $I$ of $\Pi, \rho_{A}(I) \leq r$. In such case, $A$ is called an $r$-approximation algorithm of $\Pi$.

Given two problems $\Pi_{1}$ and $\Pi_{2}$, we reduce $\Pi_{1}$ to $\Pi_{2}$ by providing two polynomial-time computable functions $f$ and $g$ such that $f$ transforms any instance $I_{1}$ in $\Pi_{1}$ into an instance $I_{2}=f\left(I_{1}\right)$ in $\Pi_{2}$, and $g$ transforms any feasible solution $S_{2}$ of this $I_{2}$ into a feasible solution $g\left(S_{2}\right)$ of the initial instance $I_{1}$.

We say that the reduction is strict $\left(\Pi_{1} \leq_{S} \Pi_{2}\right)$ if in addition, for any instance $I_{1} \in \mathcal{I}_{\Pi_{1}}$ and any feasible solution $S_{2}$ of $f\left(I_{1}\right)$, the performance ratios satisfy

$$
\rho_{\Pi_{1}}\left(I_{1}, g\left(S_{2}\right)\right) \leq \rho_{\Pi_{2}}\left(f\left(I_{1}\right), S_{2}\right) .
$$

Given such a strict reduction, the optimal solution of $f\left(I_{1}\right)$ would lead to an optimal solution of $I_{1}$, and furthermore, any feasible solution of $f\left(I_{1}\right)$ would lead to a feasible solution of $I_{1}$ with better performance ratio. It is straightforward to see that an $r$ approximation algorithm of $\Pi_{2}$ would lead to an $r$-approximation algorithm of $\Pi_{1}$. Therefore, strict reduction preserves the membership of APX. The following lemma will be useful for our inapproximability proof.

Lemma 2.3.1 If $\Pi_{1} \leq_{S} \Pi_{2}$ and $\Pi_{1} \notin \mathrm{APX}$, then $\Pi_{2} \notin \mathrm{APX}$.

In other words, if $\Pi_{1}$ is strictly reducible to $\Pi_{2}$ and cannot be approximated within any constant factor, neither can $\Pi_{2}$.

\section{Related Work}

Researchers have been interested in localizing one-dimensional homology classes with the minimal volume cycle, namely, the shortest representative cycle. Using Dijkstra's shortest path algorithm, Erickson and Whittlesey [19] computed the shortest homology basis of 2-manifolds, namely, the one-dimensional homology cycle basis whose elements have the minimal total volume. Dey et al. [16] provided a polynomial-time algorithm to compute such basis when the input is a general simplicial complex.

These polynomial-time algorithms cannot localize an arbitrarily given class. To fill this void, Chambers et al. [7] (see [18] for a recent improvement) devised an algorithm to localize a given class when the input is a 2-manifold, with or without boundary. Their method precomputes the shortest representative cycles of all $2^{\beta_{1}}-1$ nontrivial classes and thus, is exponential in the one-dimensional Betti number $\beta_{1}$.

It has been demonstrated that when $\beta_{1}=\Theta(n)$, localizing a given one-dimensional class with its shortest cycle is NP-hard, both in the cases that the topological space is a general complex [8] and a 2-manifold [7].

Due to the difficulties in localizing with the minimal volume criterion, researchers have focused on other criteria or heuristics. Some have computed onedimensional cycles closely related to handles which are much more meaningful in 
low-dimensional applications such as graphics and CAD. Guskov and Wood [21, 29] detected small handles of a 2-manifold using the Reeb graph of the manifold. Given a 2-manifold embedded in $S^{3}$, Dey et al. [14] computed these handle-related cycles by computing the deformation retractions of the two components of the embedding space bounded by the given 2-manifold. A recent extension [15] improved their result based on geometric heuristics and persistent homology. Their work facilitates handle detection in real applications.

All of the aforementioned works are restricted to one-dimensional homology. Zomorodian and Carlsson [30] took a different approach to solving the localization problem for general dimension. Their method starts with a topological space and a cover, which is a set of spaces whose union contains the original space. They computed a homology basis and localized classes of it, using tools from algebraic topology and persistent homology. However, both the quality of the localization and the complexity of the algorithm depend strongly on the choice of cover; there is, as yet, no suggestion of a canonical cover.

Chen and Freedman [10] presented a polynomial-time algorithm for localizing a homology class of general dimension with the minimal radius cycle. Their algorithm can also compute a homology cycle basis with the minimal total radius. The cycle with the minimal radius, however, may be quite complicated in terms of geometry. See Sect. 5 for detailed discussion.

In terms of homology over other fields, Chambers et al. [6] addressed the localization problem of one-dimensional homology over other fields by formulating a maximization problem. They view a 1-chain as a flow of the 1-skeleton of a simplicial complex. The localization problem is formalized as finding a maximal flow homologous to a given flow under a given constraint of the edge capacities. Two 1-chains are homologous if their difference is a 1-boundary. Their algorithm is exponential in $\beta_{1}$ for real coefficients and $O\left(\beta_{1}^{7} n \log ^{2} n \log ^{2} C\right)$ for integer coefficients, where $C$ is the total sum of all the edge capacities.

Dey et al. [13] showed that the localization problem with minimal volume is polynomial-time solvable for a class of spaces when the homology is defined over the integers $\mathbb{Z}$. In particular, they showed that when the input space is an orientable $(d+1)$-dimensional manifold and the homology class in question is $d$-dimensional, the localization problem is polynomial over $\mathbb{Z}$, despite its being NP-hard over the $\mathbb{Z}_{2}$ field [7].

\section{Localization with Volume}

\subsection{Problem Formalization and a List of Existing Results}

Given a cost function defined on all the $d$-cycles, cost $: Z_{d}(K) \rightarrow \mathbb{R}$, we formalize the localization problem as a combinatorial optimization problem.

Problem 4.1.1 (Localizing Homology)

INPUT: a simplicial complex $K$ with size $n$, a d-dimensional nontrivial homology class $h=\left[z_{0}\right], 0 \leq d \leq \operatorname{dim}(K)$ 
OUTPUT: a cycle $z \in h$

MINIMIZE: $\operatorname{cost}(z)$

In this section, we use volume as the cost function. Other cost functions would be discussed in Sect. 5.

Definition 4.1.2 (Volume) The volume of a cycle is the number of its simplices,

$$
\operatorname{vol}(z)=\operatorname{card}(z)
$$

For example, the volume of a 1-cycle, a 2-cycle and a 3-cycle are the numbers of their edges, triangles and tetrahedra, respectively. The cycle with the smallest volume, denoted as $z_{v}$, agrees intuitively with the notion of a "well-localized" cycle. For convenience, we denote LocHomVol as the problem of localizing a homology class with its minimal volume cycle, $z_{v}$.

More generally, we can extend the volume definition to be the sum of the weights assigned to simplices of the cycle, given an arbitrary weight function $w: K \rightarrow \mathbb{R}$, defined on all the simplices of $K$; formally,

$$
\operatorname{vol}_{G}(z)=\sum_{\sigma \in z} w(\sigma) .
$$

Computing $z_{v}$ using this general volume definition is at least as hard as using Definition 4.1.2, which is in fact a special case $(w(\sigma)=1 \forall \sigma \in K)$. Therefore, we will only treat the unweighted volume function.

There are two other variations, which are supposed to be easier than LocHomVol, namely, computing a nonbounding cycle with the minimal volume and computing a homology cycle basis with the minimal total volume; formally:

Problem 4.1.3 (Min-Vol Nonbounding Cycle)

INPUT: a simplicial complex $K$ with size $n$

OUTPUT: a nonbounding $d$-cycle $z, 0 \leq d \leq \operatorname{dim}(K)$

MINIMIZE: $\operatorname{vol}(z)$

Problem 4.1.4 (Min-Vol Basis)

INPUT: a simplicial complex $K$ with size $n$

OUTPUT: a homology cycle basis $\left\{z_{1}, z_{2}, \ldots, z_{\beta_{d}}\right\}, 0 \leq d \leq \operatorname{dim}(K)$

MINIMIZE: $\quad \sum_{i=1}^{\beta_{d}} \operatorname{vol}\left(z_{i}\right)$

We name these two problems MinVolNBCyc and MinVolBasis, respectively. For clarity, we show in Fig. 2 the difference between MinVolBasis and LocHomVol. The former is easier than the latter.

There are some existing hardness results, when the homology classes in question are one-dimensional.

- When $\beta_{1}=\Theta(n)$, LocHomVol is proved to be NP-hard by polynomial reductions from a special case of MAX-2SAT [8] and MIN-CUT with negative edge weights [7]. 
Fig. 2 A rectangle with two holes. The output of MinVolBasis is the two blue 1-cycles, $z_{1}$ and $z_{2}$. However, the minimal volume cycle in $\left[z_{1}+z_{2}\right]$ is the red cycle, which cannot be computed using the optimal homology cycle basis

Table 1 Existing results for one-dimensional homology

\begin{tabular}{ll}
\hline LocHomVol, $\beta_{1}=\Theta(n)$ & NP-hard \\
LocHomVol, $\beta_{1}=O(1)$, 2-manifolds & Polynomial \\
LocHomVol, $\beta_{1}=O(1)$, general complexes & Unknown \\
MinVolNBCyc & Polynomial \\
MinVolBasis & Polynomial \\
\hline
\end{tabular}

- Chambers et al. [7] provided a polynomial-time algorithm for LocHomVol when $\beta_{1}$ is fixed (the algorithm is improved in [18]). The algorithm computes the shortest representative cycle for each of the $2^{\beta_{1}}-1$ nontrivial classes. This work is restricted to triangulations of 2-manifolds with or without boundaries. The problem remains open when the input is a general simplicial complex.

- Erickson and Whittlesey [19] devised a polynomial-time algorithm for MinVolBasis, even when $\beta_{1}=\Theta(n)$. This work is restricted to triangulations of 2-manifolds. Dey et al. [16] provided a polynomial-time algorithm when the input is a general simplicial complex.

We summarize these results in Table 1.

All these existing results are about one-dimensional homology. In this paper, we will study whether LocHomVol is difficult in general dimension and, more importantly, how difficult it is.

The existing results suggest that the localization problem might be easier if we assume fixed Betti number, or if we compute MinVolNBCyc or MinVolBasis instead. Therefore, we would also like to find out how difficult these problems could be. We prove the inapproximability of a special case of MinVolNBCyc, namely, when $\beta_{d}=1$, which in turn shows that all the problems we are interested in are NP-hard to approximate when the homology is two-dimensional or higher.

For the sake of clarity, we list all the new results as follows.

- When the homology in question is one-dimensional or higher and the Betti number is $\Theta(n)$, it is NP-hard to approximate LocHomVol within any constant factor (Theorem 4.2.4).

- When the homology in question is two-dimensional or higher, we prove that MinVolNBCyc is NP-hard to approximate within any constant factor (Theorem 4.3.3). So do LocHomVol with $\beta_{d}=O(1)$ and MinVolBasis (Corollary 4.3.4).

- A polynomial-time algorithm to compute the minimal volume nonbounding cycle for a special case where the pertinent space is embedded in $\mathbb{R}^{N}$ and the pertinent homology is $(N-1)$-dimensional. 
Fig. 3 The constructed cell complex, $T$. Two 2-cells (pipes) share four 1-cells (thickened circles), corresponding to two columns and four rows of $A$

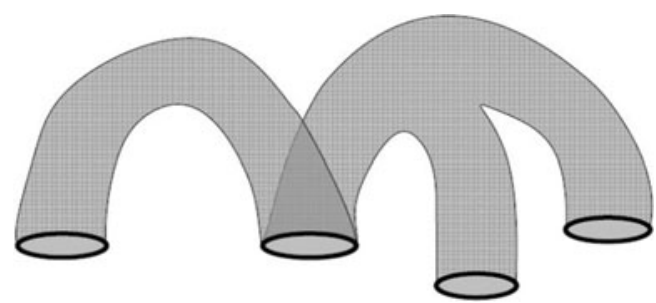

\subsection{LocHomVol is NP-hard to Approximate Within any Constant Factor}

We prove by a strict reduction from the nearest codeword problem (NearestCodeword), which cannot be approximated within any constant factor [2]. Problems used in previous reductions to LocHomVol [7, 8] have constant approximation ratios and thus cannot be used for our proof.

Problem 4.2.1 (Nearest Codeword Problem)

INPUT: an $m \times k$ generator matrix A over $\mathbb{Z}_{2}$ and a vector $y_{0} \in \mathbb{Z}_{2}^{m} \backslash \operatorname{span}(A)$

OUTPUT: a vector $y \in y_{0}+\operatorname{span}(A)$

MINIMIZE: the Hamming weight of $y$

Lemma 4.2.2 For one-dimensional homology, LocHomVol cannot be approximated within any constant factor.

Proof We prove by a strict reduction from NearestCodeword, namely,

$$
\text { NearestCodeword } \leq_{S} \text { LocHomVol. }
$$

Given an instance of NearestCodeword, namely, a generator matrix $A$ and a vector $y_{0}$, we first construct a cell complex $T$ whose two-dimensional boundary matrix is $A$. $T$ has $m$ 1-cells and $k 2$-cells corresponding to the $m$ rows and $k$ columns of $A$. Each 1 -cell is a one-dimensional cycle. Each 2-cell is a pipe with multiple openings. Note that we are abusing notation when we call $T$ a cell complex, as these cells may not be homeomorphic to closed balls. See Fig. 3 for an example with a $4 \times 2$ generator matrix

$$
A=\left[\begin{array}{ll}
1 & 0 \\
1 & 1 \\
0 & 1 \\
0 & 1
\end{array}\right] .
$$

The column space $\operatorname{span}(A)$ one-to-one corresponds to the set of boundaries of $T$. The $m$-dimensional binary vector space $\mathbb{Z}_{2}^{m}$ one-to-one corresponds to the set of 1 cycles of $T$. Therefore, NearestCodeword is identical to the problem of computing the minimal volume representative cycle of a given one-dimensional class of $T,\left[y_{0}\right]$. However, this problem, denoted as LocHomVol- $T$, is different from LocHomVol, whose input is a simplicial complex. We will prove in Lemma 4.2.3 that we can 
strictly reduce LocHomVol- $T$ to LocHom Vol by subdividing $T$ into a simplicial complex $K$. This concludes the proof.

For convenience, we introduce some notation before proving Lemma 4.2.3. We call a 1-chain $c$ a simple path if $\operatorname{card}(c)=\operatorname{card}(\operatorname{vert}(c))+1$, and there is a nonrepeating sequence of $\operatorname{vert}(c),\left(v_{1}, v_{2}, \ldots, v_{k}\right)$, such that any two consecutive vertices in the sequence is connected by an edge of $c .^{2}$ The first and last vertices are the end vertices. If we identify the two end vertices, that is, $v_{1}=v_{k}$, the chain $c$ is called a simple cycle. In this case, $\operatorname{card}(c)=\operatorname{card}(\operatorname{vert}(c))$. We extend the definition of homologous to chains. Two chains are homologous to each other if their difference is a boundary.

\section{Lemma 4.2.3}

\section{LocHomVol- $T \leq{ }_{S}$ LocHomVol.}

Proof We prove by subdividing $T$ into a simplicial complex $K$, whose size is polynomial to the size of $T$. Recall $m$ is the number of 1-cells of $T$. For each 2-cell of $T$, $\sigma$, we triangulate it as follows.

1. Cut $\sigma$ into a polygon with $2 a$ sides, where $a$ is the cardinality of $\partial \sigma$. For example, in Fig. 4, $\sigma$ is cut into an octagon.

2. Triangulate each of the $a$ sides corresponding to 1 -cells of $T$ into $t_{1}$ edges.

3. Triangulate the interior of the polygon fine enough so that for any two vertices from two nonadjacent sides of the polygon, any path connecting them has at least $t_{1} m$ edges. See Fig. 4 for the case where $\operatorname{card}(\partial \sigma)=4, t_{1}=4$ and $m=5$.

Note that the size of $K$ is polynomial to the size of $T$ and thus can be produced in polynomial time.

For convenience, we denote the triangulation of all 1-cells of $T$ as $K_{1}$, which is a subcomplex of $K$. The number of edges in $K_{1}$ is $m t_{1}$. There is a one-to-one correspondence between 1-cycles of $T$ and 1-cycles of $K_{1}$, denoted as $\phi$. For any 1-cycle of $T, y$, and its corresponding 1-cycle of $K_{1}, \phi(y)$, the ratio of their volumes is $1: t_{1}$.

Our construction provides a polynomial transformation of every instance of LocHomVol- $T,\left(T, y_{0}\right)$, into an instance of LocHomVol, $\left(K, z_{0}=\phi\left(y_{0}\right)\right)$. For any such instance and any feasible solution $z \in\left[z_{0}\right]$, we will show a polynomial-time algorithm transforming $z$ into a cycle $z^{\prime} \in\left[z_{0}\right]$ carried by $K_{1}$ and such that $\operatorname{vol}\left(z^{\prime}\right) \leq$ $\operatorname{vol}(z)$. This leads to a solution of LocHomVol- $T, \phi^{-1}\left(z^{\prime}\right)$. For convenience, we denote this solution $g(z)$.

This reduction is strict. First, the optimal solution of LocHomVol, $z_{v}$, is a cycle of $K_{1}$, whose corresponding solution of LocHomVol-T, $g\left(z_{v}\right)=\phi^{-1}\left(z_{v}\right)$, is the optimal solution of LocHomVol-T. The ratio of their volumes is $\operatorname{vol}\left(z_{v}\right): \operatorname{vol}\left(g\left(z_{v}\right)\right)=$ $t_{1}: 1$. Second, for any feasible solution $z$, the volume of its corresponding solution in

\footnotetext{
${ }^{2}$ This definition is consistent with the definition in graph theory.
} 


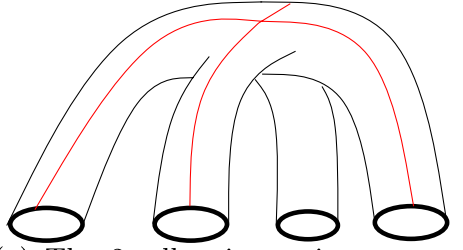

(a) The 2-cell $\sigma$ is cut into an octagon along the red curves. $\partial \sigma$ is highlighted with thickened lines.

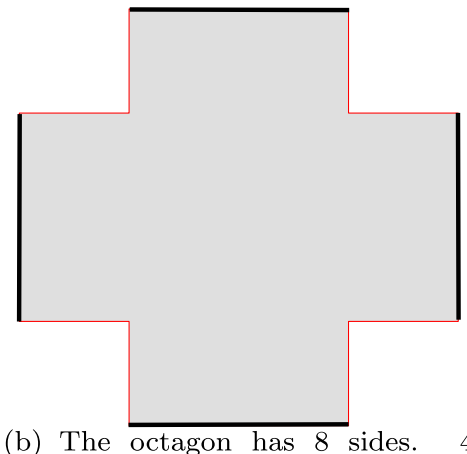

of them correspond to 1-cells of $T$. Note that the other 4 sides (drawn in red) are not drawn as straight lines.

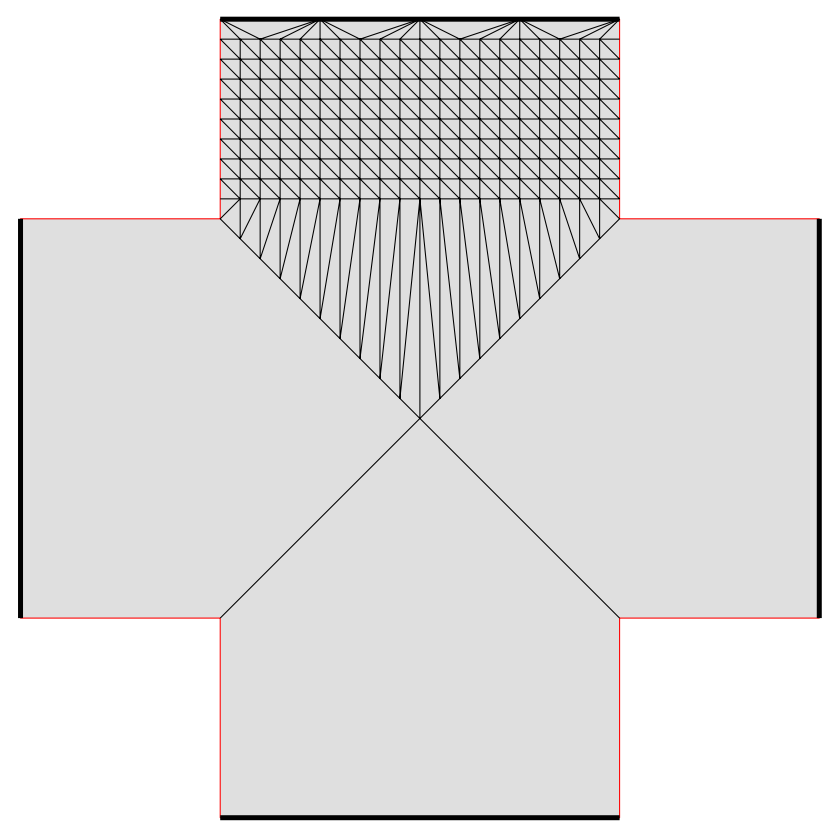

(c) A fine triangulation of the polygon. For simplicity, we only draw $1 / 4$ of the triangulation. The size of this triangulate is polynomial to $m$ and $t_{1}$.

Fig. 4 The triangulation of a 2-cell of $T$, $\sigma$, with $\operatorname{card}(\partial \sigma)=4, t_{1}=4$ and $m=5$

\section{LocHomVol- $T$ is}

$$
\begin{aligned}
& \operatorname{vol}(g(z))=\operatorname{vol}\left(\phi^{-1}\left(z^{\prime}\right)\right)=\frac{1}{t_{1}} \operatorname{vol}\left(z^{\prime}\right) \leq \frac{1}{t_{1}} \operatorname{vol}(z), \\
& \text { and therefore, } \frac{\operatorname{vol}(g(z))}{\operatorname{vol}\left(g\left(z_{v}\right)\right)} \leq \frac{\operatorname{vol}(z)}{\operatorname{vol}\left(z_{v}\right)} .
\end{aligned}
$$




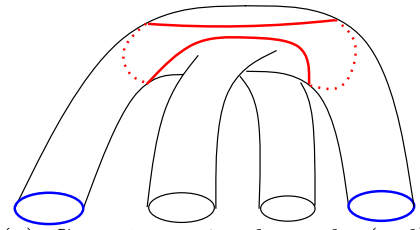

(a) Case 1: a simple cycle (red) is homologous to a 1-cycle (blue) carried by $K_{1}$. Note the latter cycle has two components.

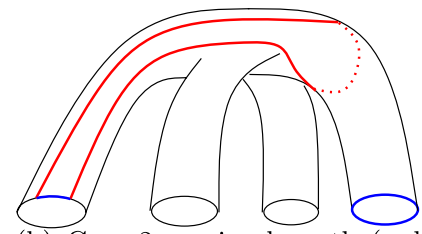

(b) Case 2: a simple path (red) whose end vertices are from the triangulation of a same 1-cell is homologous to a 1-chain (blue) carried by $K_{1}$.

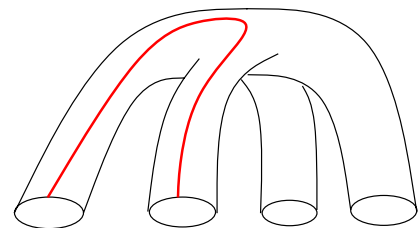

(c) Case 3: a simple path (red) connecting vertices from the triangulation of two 1-cells is at least $m t_{1}$ long.

Fig. 5 Different cases for generating $z^{\prime}$

This guarantees Inequality (1) and thus the strictness of the reduction.

To conclude this proof, we show that for any given cycle of $K, z \in\left[z_{0}\right]$ with $z_{0}$ carried by $K_{1}$, there is a polynomial-time algorithm which computes a cycle $z^{\prime} \in\left[z_{0}\right]$ carried by $K_{1}$ and such that $\operatorname{vol}\left(z^{\prime}\right) \leq \operatorname{vol}(z)$. We partition $z$ into simple cycles and simple paths by finding all repeating vertices and vertices of $K_{1}$. Each simple cycle has no vertex from $K_{1}$. Each simple path has no vertices from $K_{1}$ except for the two end vertices. Next, we deal with these simple cycles and simple paths one by one. For each simple cycle or simple path, which is carried by the triangulation of one 2-cell $\sigma$ of $T$, we find a 1 -chain homologous to it and carried by $K_{1}$. The new chain has a smaller or equal volume.

There are three cases. Recall that $\phi$ maps a 1-chain of $T$ to its subdivision.

1. If a simple cycle is trivial, replace it with an empty chain. Otherwise, this cycle is homologous to a cycle carried by the triangulation of $\partial \sigma, \phi(\partial \sigma) \subseteq K_{1}$. The latter cycle has a smaller or equal volume, due to the way we subdivide $\sigma$. See Fig. 5(a) for an example.

2. For a simple path whose both end vertices are from the triangulation of a same 1-cell of $T, \tau \in \partial \sigma$, it is homologous to a path connecting the two end vertices within $\phi(\tau)$ plus cycles which are triangulations of other cells of $\partial \sigma$. The latter chain has a smaller or equal volume, due to the way we subdivide $\sigma$. See Fig. 5(b).

3. Suppose that it is a simple path connecting vertices from the triangulations of two different 1-cells (Fig. 5(c)). Such path has a volume of at least $m t_{1}$. In such case, we let $z^{\prime}$ be the input $z_{0}$, whose volume is not greater than $m t_{1}$ and thus not greater than $\operatorname{vol}(z)$. 
$z^{\prime}$ is computed after we transform all simple paths and simple cycles into homologous chains and cycles carried by $K_{1}$, or we let $z^{\prime}=z_{0}$ if Case 3 happens.

Lemma 4.2.2 is about one-dimensional homology. We extend the result to homology of any higher dimension.

Theorem 4.2.4 For any $d \geq 1$, LocHomVol for $d$-dimensional homology cannot be approximated within any constant factor.

Proof We show that when $d \geq 2$, LocHomVol for $(d-1)$-dimensional homology can be strictly reduced to LocHomVol for $d$-dimensional homology, namely, LocHomVol $_{d-1} \leq_{S}$ LocHomVol $_{d}$. Together with Lemma 4.2.2, the theorem is proved.

Next, we explain the reduction. Given a simplicial complex of $\operatorname{LocHomVol}_{d-1}$, we build a suspension of it, namely, two cones of the complex glued together at their base [28]. There is a one-to-one correspondence between the $(d-1)$-dimensional cycle group of the original complex and the $d$-dimensional cycle group of the new complex. This correspondence also works for the boundary groups. Since the volume of each $(d-1)$-cycle is $1 / 2$ of the volume of its corresponding $d$-cycle, this is a strict reduction.

Restriction to a manifold A natural question is whether the localization problem could be made easier if we restrict the input to be the triangulation of a manifold. We could modify Lemma 4.2.2 and its proof to accommodate this manifold assumption. Specifically, we embed the cell complex $T$ in $\mathbb{R}^{N}$. By thickening the underlying space of $T$ and taking its boundary as a new topological space, we get an $(N-1)$ manifold (one less dimension than the ambient space). This manifold can be triangulated in a similar way as we triangulate $T$ and thus leads to the inapproximability of LocHomVol for one-dimensional homology when the input is the triangulation of an $(N-1)$-manifold. We omit a formal description of such reduction. See Fig. 4(b) for an example when $T$ is one-dimensional and $N=2$.

A classical result suggests that we can embed the two-dimensional cell complex $T$ in $\mathbb{R}^{5}$. Using an analog of book embedding an arbitrary graph in $\mathbb{R}^{3}$ [27], we can embed $T$ in $\mathbb{R}^{4}$. Therefore, we prove the problem is NP-hard to approximate for onedimensional homology when the input is the triangulation of a 3-manifold. This raises the open question that whether localizing a one-dimensional class of a 2-manifold is NP-hard to approximate (it has already been proved to be NP-hard to compute).

A similar argument can be applied to other problems we will discuss in the next section, except that in Lemma 4.3.1, the relevant homology is two-dimensional, the cell complex $T$ is three-dimensional, and the manifold is four-dimensional.

\subsection{MinVolNBCyc is NP-hard to Approximate Within any Constant Factor}

In the previous section, the simplicial complex we constructed for LocHomVol has $\Theta(n)$ Betti number. It has been revealed for one-dimensional homology that

- MinVolNBCyc and MinVolBasis can be solved in polynomial time, and 


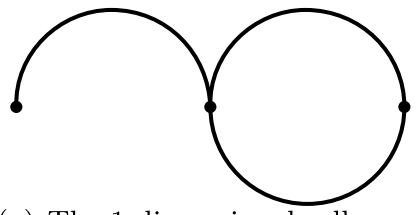

(a) The 1-dimensional cell complex $T$, embedded in $\mathbb{R}^{2}$.

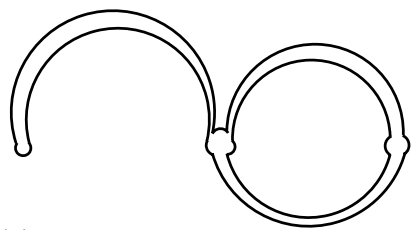

(c) Take the boundary of the thickened $T$ as the new topological space, which is a 1-manifold.

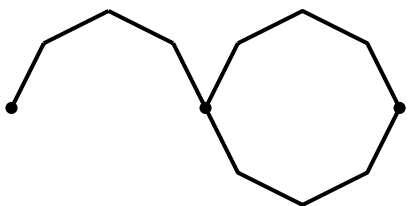

(b) Triangulate $T$ into a simplicial complex, according to the scheme in Lemma 4.2.3.

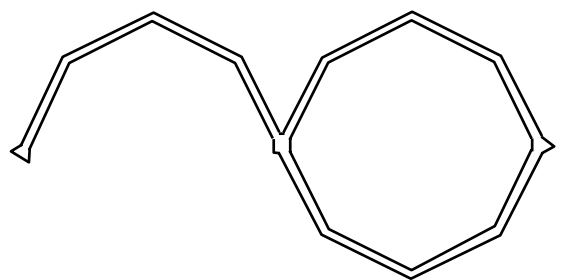

(d) Triangulate the 1-manifold according to the way $T$ is triangulated. The triangulation is polynomial.

Fig. 6 An example of modifying proof of Lemma 4.2.3 to accommodate the manifold assumption

- LocHomVol with $\beta_{1}=O(1)$ can be solved in polynomial time when the input is the triangulation of a 2-manifold, with or without boundary.

This raises the question of whether these three problems are hard for homology of dimension two or higher. Our main result in this section is the inapproximability proof of a special case of MinVolNBCyc (Theorem 4.3.3). This trivially leads to the inapproximability of all the aforementioned problems (Corollary 4.3.4).

Lemma 4.3.1 For 2-dimensional homology, even when $\beta_{2}=1$, MinVolNBCyc is $N P$-hard to approximate within any constant factor.

Proof We prove by a strict reduction from NearestCodeword, namely,

$$
\text { NearestCodeword } \leq{ }_{S} \text { MinVolNBCyc. }
$$

Given an instance of NearestCodeword, we consider the generator matrix $C=$ $\left[A, y_{0}\right]$ and its parity-check matrix $C^{\perp}$ (the dimension is $\left.(m-k-1) \times m\right)$. Following a scheme similar to Lemma 4.2.2 (illustrated in Fig. 3), we construct a cell complex $T_{2}$ using $C^{\perp}$ as the two-dimensional boundary matrix. $T_{2}$ has $m-k-11$-cells and $m$ 2-cells. There is a one-to-one correspondence between the two-dimensional cycle group of $T_{2}$ and nullspace $\left(C^{\perp}\right)=\operatorname{span}(C)$. This cycle group has dimension $k+1$ and is spanned by the column vectors of $A$ and $y_{0}$.

Next, for each column vector of $A$, we seal the corresponding 2-cycle in $T_{2}$ with a 3-cell. $T_{2}$ is the 2-skeleton of the augmented complex, which is denoted as $T$. The one and only nontrivial two-dimensional homology class of $T$ is identical to the coset $y_{0}+\operatorname{span}(A)$. Finding the smallest volume nonbounding 2-cycle of $T$, denoted as MinVolNBCyc- $T$, is equal to finding the minimal Hamming weight vector 
in this coset and thus equal to solving NearestCodeword. It suffices to show that MinVolNBCyc- $T$ can be strictly reduced to MinVolNBCyc by subdividing $T$.

In order to triangulate $T$ into a simplicial complex $K$, we first subdivide the 2skeleton $T_{2}$ into a simplicial complex $K_{2}$, in which all 2-cells are triangulated into the same number of triangles (say, $t_{2}$ ). There is a one-to-one correspondence between the two-dimensional cycle groups $Z_{2}\left(K_{2}\right)$ and $Z_{2}\left(T_{2}\right)=Z_{2}(T)$. The volume of each 2-cycle of $K_{2}$ is $t_{2}$ times that of its corresponding cycle.

Next, while keeping $K_{2}$ intact, we triangulate interior of the 3-cells fine enough so that for any nonbounding 2-cycle of $K, z \in\left[z_{0}\right]$ with $z_{0}$ carried by $K_{2}$, we can always find in polynomial time a nonbounding 2-cycle of $K_{2}, z^{\prime} \in\left[z_{0}\right]$, with a smaller or equal volume. (This is similar to the triangulation strategy in Lemma 4.2.3.) Due to the one-to-one correspondence between $Z_{2}\left(K_{2}\right)$ and $Z_{2}(T)$ and the $t_{2}: 1$ ratio of their volumes, we have a strict reduction from MinVolNBCyc- $T$ to MinVolNBCyc.

Remark 4.3.2 Whereas $\beta_{2}$ and $\beta_{3}$ of the constructed $K$ are 1 and 0 , respectively, the one-dimensional Betti number $\beta_{1}$ could be linear in the size of $K$. However, we can remedy this by computing an arbitrary one-dimensional homology cycle basis and seal all its elements with additional triangles. This will not influence the reduction. This way, we prove the inapproximability for complexes with bounded Betti numbers of all dimensions.

Similar to Theorem 4.2.4, we can extend the result to any higher dimension by a suspension-building-based strict reduction of any MinVolNBCyc problem for $(d-$ 1)-dimensional homology to that for the $d$-dimensional homology.

Theorem 4.3.3 Even when the relevant Betti number is 1, MinVolNBCyc is NP-hard to approximate within any constant factor for homology of dimension two or higher.

So far the inapproximability proof is for MinVolNBCyc with $\beta_{d}=1$. This trivially leads to the inapproximability of the general MinVolNBCyc. Furthermore, we extend the inapproximability to the other two problems.

Corollary 4.3.4 For homology of dimension two or higher, the following problems are NP-hard to approximate within any constant factor:

1. MinVolBasis;

2. LocHomVol with fixed Betti number.

Proof We show that the special case MinVolNBCyc can be computed in polynomial time from the output of the other two problems. This leads to the inapproximability.

Given the output of MinVolBasis, the homology cycle basis with the minimal total volume, the minimal volume nonbounding cycle is in this basis.

For LocHomVol with fixed Betti number, we enumerate all nontrivial classes and find their minimal volume representatives. The minimal volume nonbounding cycle is one of those representatives. 


\subsection{A Polynomial Special Case}

There is, however, a special case in which MinVolNBCyc can be computed in polynomial time, even with linear Betti number: when $K$ is an $N$-dimensional complex embedded in $\mathbb{R}^{N}$, and the pertinent nonbounding cycle is $(N-1)$-dimensional. In this section, we provide a polynomial-time algorithm, inspired by [7, 22]. It is not hard to generalize this algorithm to MinVolBasis and LocHomVol.

We add new $N$-cells to $K$ to get a new complex $K^{\prime}$, whose underlying space is $\mathbb{R}^{N}$. Each new cell covers one component of $\mathbb{R}^{N} \backslash|K|$. There are $\beta_{N-1}+1$ new cells, one of which covers the infinity component. The boundary of each new cell is one component of the $(N-1)$-dimensional boundary of $K$. Here we are abusing notation again as the new cells may not be homeomorphic to closed balls.

We use the MIN-CUT algorithm on the dual graphs to solve the problem. The dual graph $G$ of $K$ is a subgraph of the dual $G^{\prime}$ of $K^{\prime}$. Denote vertex sets of $G$ and $G^{\prime}$ as $V$ and $V^{\prime}$, respectively. The set of new vertices $V^{\prime} \backslash V$ is dual to the set of new $N$-cells. See Fig. 7 for an example when $N=2$.

We call a cycle minimal if none of its nonempty subsets is a cycle. We denote by $C\left(G^{\prime}, G\right)$ the set of minimal edge cuts (cuts whose subsets are not cuts) of $G^{\prime}$ which cut $G^{\prime}$ into two partitions each of which contains at least one vertex of $V^{\prime} \backslash V$. There is a one-to-one correspondence between the set of minimal nonbounding $(N-1)$-cycles of $K$ and the set of cuts $C\left(G^{\prime}, G\right)$. The volume of each cycle is equal to the cardinality of its corresponding cut. As the nonbounding $(N-1)$-cycle with the smallest volume has to be one of the minimal cycles, it can be computed by computing the cut in $C\left(G^{\prime}, G\right)$ with the smallest cardinality.

To compute the minimal cardinality cut in $C\left(G^{\prime}, G\right)$, we enumerate all pairs of vertices, $\left(v_{1}, v_{2}\right) \in\left(V^{\prime} \backslash V\right) \times\left(V^{\prime} \backslash V\right)$. Compute the minimal $\left(v_{1}-v_{2}\right)$-cut for each pair. The one with the smallest cardinality is the desired one.

Since the cardinality of $V^{\prime} \backslash V$ is $\beta_{N-1}+1$, the complexity of this algorithm is $O\left(\beta_{N-1}^{2} f(n)\right)$ where $n$ is the size of the simplicial complex, and $f(n)$ is the complexity of the MIN-CUT algorithm. Using MIN-CUT algorithms whose complexity is $O\left(n^{2} \log n\right)$, the whole algorithm has complexity $O\left(\beta_{N-1}^{2} n^{2} \log n\right)$.

Remark 4.4.1 The idea can be carried over to the case of a weighted volume function, but only if the weight function is nonnegative.

Fig. 7 A two-dimensional simplicial complex embedded in $\mathbb{R}^{2}$. The dual graph $G$ and $G^{\prime}$ are drawn in solid lines and vertices. Their difference $G^{\prime} \backslash G$ includes vertices $p_{1}, p_{2}, p_{3}$ and their incident edges

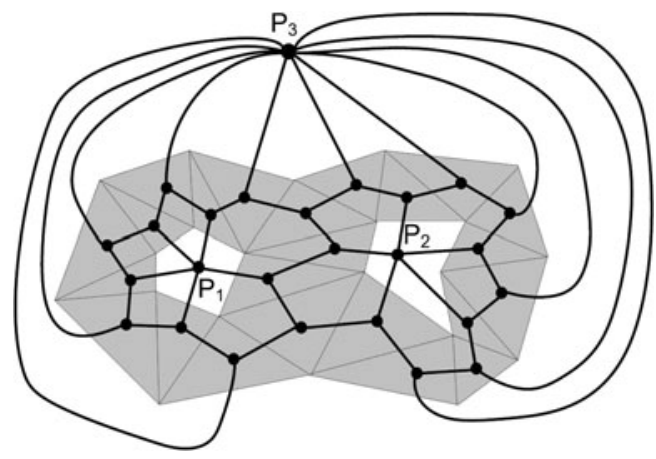




\section{Localization with Other Geometric Criteria}

\subsection{Diameter}

When LocHomVol is proved to be NP-hard to approximate, we resort to discrete geodesic distance related cost functions, diameter and radius.

Definition 5.1.1 (Diameter) The diameter of a cycle is the diameter of its vertex set, $\operatorname{diam}(z)=\operatorname{diam}(\operatorname{vert}(z))$, in which the diameter of a set of vertices is the maximal discrete geodesic distance between them; formally,

$$
\operatorname{diam}(S)=\max _{p, q \in S} \operatorname{dist}(q)
$$

Intuitively, a representative cycle of $h$ with the minimal diameter, denoted $z_{d}$, is the cycle whose vertices are as close to each other as possible. The intuition will be further illustrated in Sect. 5.2 by comparison against other criteria. We prove in Theorem 5.1.4 that computing $z_{d}$ of $h$ is NP-hard, by reduction from a special case of the NP-hard Multiple-Choice Cover Problem (MCCP) of Arkin and Hassin [1]. The theorem has been stated in our previous paper [9]. But the proof has not been published.

Remark 5.1.2 We do not address the approximability of LocHomDiam, as we realize that $z_{d}$ suffers from a "wiggling problem" and consequently may be geometrically complex (see Sect. 5.2). However, it is not hard to see that the reduction in Theorem 5.1.4 is strict, which implies that LocHomDiam cannot be approximated any better than this special case of MCCP, which cannot be approximated within $2-\epsilon$ for any $\epsilon>0$, though we do not establish this formally.

Problem 5.1.3 (Multiple-Choice Cover Problem)

INPUT: $\quad$ a set of vertices, $V=\left\{v_{1}, v_{2}, \ldots, v_{n}\right\}$; a distance function dist : $V \times V \rightarrow$ $\mathbb{R}^{+}$satisfying triangular inequality; Disjoint subsets of $V, S_{1}, S_{2}, \ldots, S_{m}$, such that $\bigcup_{i=1}^{m} S_{i}=V$

OUTPUT: a cover $C \subseteq V$ containing one and exactly one vertex from each subset $S_{i}$

MINIMIZE: $\operatorname{diam}(C)$

Note that the original MCCP problem of Arkin and Hassin only requires the cover to have nonempty intersection with each subset $S_{i}$. We revise the problem to facilitate our proof, without influencing the NP-hardness. The reason is that the optimal result of the revised problem is clearly an optimal result of the original problem.

Theorem 5.1.4 LocHomDiam is NP-hard to compute.

Proof We present a polynomial-time algorithm transforming an input of MCCP into an input of LocHomDiam. Later we will show that the solution of LocHomDiam gives us the solution of MCCP. As part of the input of LocHomDiam, the constructed 
simplicial complex $K$ consists of $m$ tubes $T_{1}, \ldots, T_{m}$ and extra edges connecting vertices.

We first embed the vertex set $V$ in any metric space preserving the pairwise distance $\operatorname{dist}(\cdot, \cdot)$. Without loss of generality, for ease of explanation, we assume that $V$ is embedded in the Euclidean plane $\mathbb{R}^{2}$. For each vertex subset $S_{i}$, we find a simple path in $\mathbb{R}^{2}$, going through each vertex of $S_{i}$ once without self-intersection, $\xi_{i}=\left(v_{1}, v_{2}, \ldots, v_{\text {card }\left(S_{i}\right)}\right)$, which contains card $\left(S_{i}\right)-1$ edges. The edge lengths are the same as the distances between corresponding vertices. See Fig. 8(a). We construct a slender threadlike tube $T_{i}$, which carries the path $\xi_{i} . T_{i}$ has $\left(3 \operatorname{card}\left(S_{i}\right)\right)$ vertices, $S_{i} \cup S_{i}^{\prime} \cup S_{i}^{\prime \prime}$, where

$$
S_{i}^{\prime}=\left\{v_{1}^{\prime}, v_{2}^{\prime}, \ldots, v_{\operatorname{card}\left(S_{i}\right)}^{\prime}\right\} \quad \text { and } \quad S_{i}^{\prime \prime}=\left\{v_{1}^{\prime \prime}, v_{2}^{\prime \prime}, \ldots, v_{\operatorname{card}\left(S_{i}\right)}^{\prime \prime}\right\} .
$$

For any $j, v_{j}^{\prime}$ and $v_{j}^{\prime \prime}$ lie very close to $v_{j}$. Corresponding to the $\operatorname{card}\left(S_{i}\right)-1$ edges in $\xi_{i}, T_{i}$ consists of card $\left(S_{i}\right)-1$ triangular cylinders concatenated together. By a triangular cylinder we mean the surface of a 3-prism with the two end triangles missing. To facilitate the concatenation, corresponding edges of the end triangles may not be parallel to each other, as in a standard 3-prism. Each edge $v_{j} v_{j+1}$ corresponds to a triangular cylinder with vertices $v_{j}, v_{j}^{\prime}, v_{j}^{\prime \prime}, v_{j+1}, v_{j+1}^{\prime}$ and $v_{j+1}^{\prime \prime}$. In the triangular cylinder, the short edges are very short, say, no longer than $\epsilon$. The long edges have the length similar to the length of edge $v_{j} v_{j+1}$. See Fig. 8(b) for one such triangular cylinder.

We construct the simplicial complex $K$ as follows. For any $i, T_{i} \subseteq K$. For any two vertices $v_{1}, v_{2} \in V$, if they are not neighbors, add an edge connecting them, whose length is their Euclidean distance in the Euclidean plane $\mathbb{R}^{2}$. See Fig. 8(c) for the

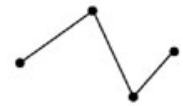

$\mathrm{S}_{1}$

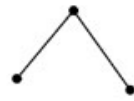

$\mathrm{S}_{2}$

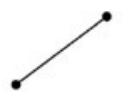

$\mathrm{S}_{3}$

(a) An input of MCCP: 3 disjoint vertex subsets in Euclidean plane, $S_{1}, S_{2}$ and $S_{3}$. The simple paths, $\xi_{1}, \xi_{2}$ and $\xi_{3}$, are also shown, although they are not part of the input.

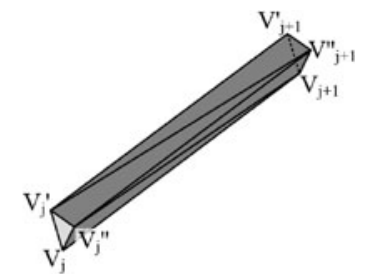

(b) A triangular cylinder corresponding to the edge $v_{j} v_{j+1}$
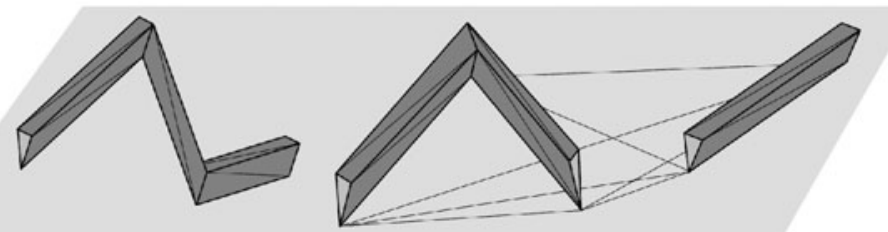

(c) The constructed simplicial complex $K$. For ease of presentation, we only show the new edges connecting vertices in $S_{2} \cup S_{3}$; however, note that there are other edges in $K$ which are omitted in this figure.

Fig. 8 Explanation of Theorem 5.1.4 proof 
complex constructed from the input in Fig. 8(a). Note that although in the figure, the embedding of $K$ in $\mathbb{R}^{3}$ has self-intersection, the simplicial complex $K$ can be embedded in Euclidean space of higher dimension, as we did in previous proofs.

For the constructed complex $K$, we use LocHomDiam to localize the onedimensional class $\sum_{i=1}^{m} h_{i}$, where $h_{i}$ is the only one-dimensional class carried by the tube $T_{i}$. We need a cycle to represent it as the input for LocHomDiam. We use $z_{0}=\sum_{i=1}^{m} z_{i_{0}}$, where $z_{i_{0}}$ is the 1-cycle whose vertices are $v_{i_{0}}, v_{i_{0}}^{\prime}$ and $v_{i_{0}}^{\prime \prime}$, in which $v_{i_{0}}$ is an arbitrary vertex in $S_{i}$.

Next, we construct a cover $C$ from the solution of LocHomDiam, $z$, and show that $C$ is the solution of MCCP. We construct an intermediate vertex set $C_{0} \subseteq V$ as follows. A vertex $v$ belongs to $C_{0}$ if and only if any of $v_{i}, v_{i}^{\prime}$ and $v_{i}^{\prime \prime}$ belongs to the vertex set of $z, \operatorname{vert}(z)$. The solution $z$ is in the form $\sum_{i=1}^{m} z_{i}$, where $z_{i}$ represents class $h_{i}$. Therefore, $C_{0}$ has nonempty intersection with each vertex set $S_{i}$. We compute the cover $C$ by picking one vertex from each $S_{i} \cap C_{0}$.

Within the simplicial complex,

$$
\operatorname{diam}(C)=\operatorname{diam}\left(C_{0}\right) \quad \text { and } \quad\left|\operatorname{diam}\left(C_{0}\right)-\operatorname{diam}(z)\right| \leq 2 \epsilon .
$$

Furthermore, $C$ has the same diameter in the simplicial complex $K$ and in the Euclidean plane, $\mathbb{R}^{2}$. Since $\epsilon$ is arbitrarily small, we can see that $C$ is the cover with the minimal diameter in the Euclidean plane and thus, is the solution of MCCP.

\subsection{Radius}

Another option for the cost function is radius.

Definition 5.2.1 (Radius) The radius of a cycle is the radius of the smallest geodesic ball carrying it; formally,

$$
\operatorname{rad}(z)=\min _{p \in \operatorname{vert}(K), z \subseteq B_{p}^{r}} r .
$$

Given a homology class, the representative cycle with the minimal radius, denoted as $z_{r}$, is the same as the localized cycle defined in our previous work [9, 10]. Intuitively, $z_{r}$ is the cycle whose vertices are as close to a vertex of $K$ as possible. Theorem 5.2.3 shows that $z_{r}$ can be computed in polynomial time.

However, in spite of its ease of computation, $z_{r}$ may not necessarily be concise in an intuitive sense. It wiggles freely inside the smallest geodesic ball carrying it. See Fig. 9(a) for example, in which we localize the only nontrivial homology class of an annulus (the light gray area). The dark gray area is the smallest geodesic ball carrying the class, whose center is $p$. Note that the geodesic ball of the annulus may not seem like a disc in the embedded Euclidean plane.

By contrast, the cycle with the minimal diameter $z_{d}$ avoids this wiggling problem in this case and is concise in an intuitive sense (Fig. 9(b)). This figure also illustrates that the radius and the diameter of a cycle are not strictly related. For the cycle $z_{r}$ in Fig. 9(a), its diameter is twice of its radius. For the cycle $z_{d}$ in Fig. 9(b), its diameter is equal to its radius.

We prove that $z_{r}$ is a 2-approximation of $z_{d}$. 


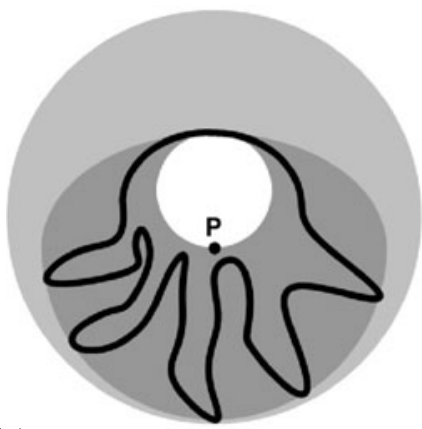

(a) The cycle with the minimal radius, $z_{r}$

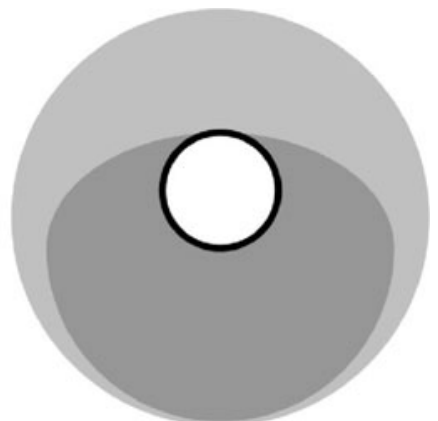

(b) The cycle with the minimal diameter, $z_{d}$.

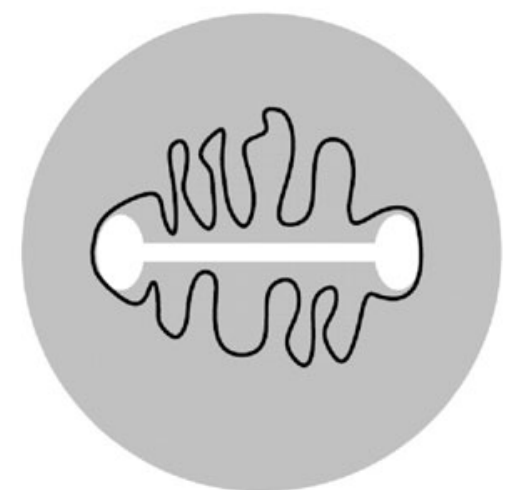

(c) A cross-section of a 3-dimensional ball with a bone shape void. $z_{d}$, which is 2-dimensional, wiggles near the middle of the bone.

Fig. 9 Cycles with the minimal radius and diameter

Theorem 5.2.2 $\operatorname{diam}\left(z_{r}\right) \leq 2 \operatorname{diam}\left(z_{d}\right)$.

Proof First, the triangle inequality of the geodesic distance suggests that for any two vertices of $z_{r}, p_{1}$ and $p_{2}$, their geodesic distance is

$$
\operatorname{dist}\left(p_{1}, p_{2}\right) \leq \operatorname{dist}\left(p_{1}, p_{0}\right)+\operatorname{dist}\left(p_{0}, p_{2}\right) \leq 2 \operatorname{rad}\left(z_{r}\right)
$$

where $p_{0}$ is the center of the smallest geodesic ball carrying the cycle $z_{r}$ and the class. This implies that the diameter of $z_{r}$ is no greater than twice of its radius.

Second, the diameter of $z_{d}$ is no less than its radius. To see this, pick a geodesic ball centered at any vertex of $z_{d}$ with radius $\operatorname{diam}\left(z_{d}\right)$. This ball carries $z_{d}$. Finally,

$$
\operatorname{diam}\left(z_{r}\right) \leq 2 \operatorname{rad}\left(z_{r}\right) \leq 2 \operatorname{rad}\left(z_{d}\right) \leq 2 \operatorname{diam}\left(z_{d}\right) .
$$

As shown in Figs. 9(a) and 9(b), this bound is a tight bound. 
However, in general, the minimal diameter cycle also suffers from the wiggling problem. In Fig. 9(c), we show an example in which the topological space is a closed three-dimensional ball with a bone shape void in the middle. The minimal-diameter 2-cycle $z_{d}$, representing the only nontrivial two-dimensional class, can freely wiggle near the middle of the bone, as the diameter is determined by the distance of the two ends of the bone. The reason for this phenomenon is that in finding the minimal diameter cycle, we minimize the maximum of all pairwise geodesic distances. It is not hard to see that $z_{d}$ does not wiggle only if for any $v \in \operatorname{vert}\left(z_{d}\right)$, its longest distance from other vertices in $z_{d}$ is close to $\operatorname{diam}\left(z_{d}\right)$.

We conclude this section by showing that $z_{r}$ can be computed in polynomial time. The proof is a short sketch of a polynomial-time algorithm of Chen and Freed$\operatorname{man}[10]$.

\section{Theorem 5.2.3 We can compute $z_{r}$ in polynomial time.}

Proof For each vertex $p$, we find the smallest geodesic ball centered at $p$ carrying any cycle in $\left[z_{0}\right]$, namely, $B_{p}^{r(p)}$, as well as the carried cycle. Iterating through all vertices $p \in \operatorname{vert}(K)$, the one with the smallest $r(p)$ gives us $z_{r}$.

To compute $B_{p}^{r(p)}$, we apply persistent homology on the complex using $f_{p}$ as the filter function. Persistent homology algorithm computes a homology cycle basis $\left\{z_{1}\right.$, $\left.z_{2}, \ldots, z_{\beta_{d}}\right\}$, sorted according to the time they enter the sublevel set. We find the smallest index $i$ so that $z_{0}$ is a linear combination of boundaries and $z_{1}, z_{2}, \ldots, z_{i}$, namely,

$$
z_{0}=\left[\partial_{d+1}, z_{1}, z_{2}, \ldots, z_{i}\right] \gamma,
$$

where $d$ is the dimension of the class in question. This can be computed by applying the persistence reduction to the matrix $\left[\partial_{d+1}, z_{1}, z_{2}, \ldots, z_{\beta_{d}}\right]$. The time $z_{i}$ enters the sublevel set is the radius $r(p)$. Replacing $\partial_{d+1}$ with 0 , we get a representative cycle of $\left[z_{0}\right]$ carried by $B_{p}^{r(p)},\left[0, z_{1}, z_{2}, \ldots, z_{i}\right] \gamma$.

The algorithm applies persistent homology algorithm $O(\operatorname{card}(\operatorname{vert}(K)))$ times. Therefore, the complexity is $O\left(n^{4}\right)$, where $n$ is the size of the simplicial complex.

\section{Conclusion}

In this paper, we have addressed the localization problem with regard to three different measures. For volume, we have proved inapproximability results. We have also proved the inapproximability of computing the nonbounding cycle with the minimal volume and computing the homology cycle basis with the minimal total volume. A special case in which a polynomial-time algorithm exists has also been discussed.

For diameter, we have proved that the localization problem is NP-hard; for radius, by contrast, we have stated a polynomial-time algorithm. Both of these two measures, however, suffer from the "wiggling problem", namely, that the output of the localization may be geometrically quite complex.

We summarize all the constructed strict reductions in this paper as follows. The arguments of a problem-specifically, the dimension $d$ of the relevant homology group and Betti numbers of the input simplicial complex — are contained in square brackets. 
- NearestCodeword $\leq_{S} \operatorname{LocHomVol-} T[d=1] \leq_{S} \operatorname{LocHomVol}[d=1]$

$$
\leq_{S} \operatorname{LocHomVol}[d \geq 1]
$$

- LocHomVol $\leq_{S}$ LocHomVol, with manifold assumption

- NearestCodeword $\leq_{S}$ MinVolNBCyc $\left[d=2, \beta_{2}=1\right]$

$$
\leq_{S} \operatorname{MinVolNBCyc}\left[d \geq 2, \beta_{d}=1\right]
$$

- MinVolNBCyc $\left[d \geq 2, \beta_{d}=1\right] \leq{ }_{S} \operatorname{MinVolBasis}[d \geq 2]$

- MinVolNBCyc $\left[d \geq 2, \beta_{d}=1\right] \leq_{S} \operatorname{LocHomVol}\left[d \geq 2, \beta_{d}=O(1)\right]$

- $\mathrm{MCCP} \leq_{S}$ LocHomDiam

An open question is whether we can use other discrete geodesic distance-related measures, besides diameter and radius, which do not suffer from the wiggling problem. For example, can we use the normalized sum of the pairwise geodesic distances? Furthermore, what if we restrict the geodesic distance to be within the cycle (rather than the entire complex)? It is conceivable that these distance-related measures might be easier to compute, as localization with the volume measure has been shown to be extremely hard.

Acknowledgements The authors thank David Cohen-Steiner and Omid Amini for constructive discussions. We thank anonymous reviewers for helpful comments.

\section{References}

1. Arkin, E.M., Hassin, R.: Minimum-diameter covering problems. Networks 36(3), 147-155 (2000)

2. Arora, S., Babai, L., Stern, J., Sweedyk, Z.: The hardness of approximate optima in lattices, codes, and systems of linear equations. J. Comput. Syst. Sci. 54(2), 317-331 (1997)

3. Ausiello, G., Paschos, V.T.: Reductions, completeness and the hardness of approximability. Eur. J. Oper. Res. 172(3), 719-739 (2006)

4. Carlsson, E., Carlsson, G., de Silva, V.: An algebraic topological method for feature identification. Int. J. Comput. Geom. Appl. 16(4), 291-314 (2006)

5. Carlsson, G.: Topology and data. Bull. Am. Math. Soc. 46(2), 255-308 (2009)

6. Chambers, E.W., Erickson, J., Nayyeri, A.: Homology flows, cohomology cuts. In: STOC (2009)

7. Chambers, E.W., Erickson, J., Nayyeri, A.: Minimum cuts and shortest homologous cycles. In: Symposium on Computational Geometry (2009)

8. Chen, C., Freedman, D.: Quantifying homology classes II: Localization and stability. CoRR, abs/0709.2512 (2007)

9. Chen, C., Freedman, D.: Quantifying homology classes. In: Proceedings of the 25th Annual Symposium on Theoretical Aspects of Computer Science, pp. 169-180 (2008)

10. Chen, C., Freedman, D.: Measuring and computing natural generators for homology groups. Comput. Geom. 43(2), 169-181 (2010)

11. Cohen-Steiner, D., Edelsbrunner, H., Morozov, D.: Vines and vineyards by updating persistence in linear time. In: Proceedings of the 22nd ACM Symposium on Computational Geometry, pp. 119-126 (2006)

12. de Silva, V., Ghrist, R.: Coverage in sensor networks via persistent homology. Algebr. Geom. Topol. 7, 339-358 (2007)

13. Dey, T.K., Hirani, A.N., Krishnamoorthy, B.: Optimal homologous cycles, total unimodularity, and linear programming. In: STOC, pp. 221-230 (2010)

14. Dey, T.K., Li, K., Sun, J.: On computing handle and tunnel loops. In: IEEE Proc. NASAGEM (2007)

15. Dey, T.K., Li, K., Sun, J., Cohen-Steiner, D.: Computing geometry-aware handle and tunnel loops in 3D models. ACM Trans. Graph. 27(3) (2008)

16. Dey, T.K., Sun, J., Wang, Y.: Approximating loops in a shortest homology basis from point data. In: Symposium on Computational Geometry, pp. 166-175 (2010)

17. Erickson, J., Har-Peled, S.: Optimally cutting a surface into a disk. Discrete Comput. Geom. 31(1), 37-59 (2004) 
18. Erickson, J., Nayyeri, A.: Minimum cuts and shortest non-separating cycles via homology covers. In: SODA (2011)

19. Erickson, J., Whittlesey, K.: Greedy optimal homotopy and homology generators. In: Proceedings of the 16th Annual ACM-SIAM Symposium on Discrete Algorithms, pp. 1038-1046 (2005)

20. Fang, Q., Gao, J., Guibas, L.: Locating and bypassing routing holes in sensor networks. In: Mobile Networks and Applications, vol. 11, pp. 187-200 (2006)

21. Guskov, I., Wood, Z.J.: Topological noise removal. In: Proceedings of the Graphics Interface 2004 Conference, pp. 19-26 (2001)

22. Kirsanov, D., Gortler, S.J.: A discrete global minimization algorithm for continuous variational problems. Technical Report TR-14-04, Harvard University (2004)

23. MacKay, D.J.C.: Information Theory, Inference \& Learning Algorithms. Cambridge University Press, Cambridge (2002)

24. Munkres, J.R.: Elements of Algebraic Topology. Addison-Wesley, Redwood (1984)

25. Niyogi, P., Smale, S., Weinberger, S.: Finding the homology of submanifolds with high confidence from random samples. Discrete Comput. Geom. 39(1), 419-441 (2008)

26. Sarkar, R., Yin, X., Gao, J., Luo, F., Gu, X.D.: Greedy routing with guaranteed delivery using Ricci flows. In: Proc. of the 8th International Symposium on Information Processing in Sensor Networks (IPSN'09), pp. 121-132 (2009)

27. Wikipedia. Book embedding. http://en.wikipedia.org/wiki/Book_embedding

28. Wikipedia. Suspension. http://en.wikipedia.org/wiki/Suspension_(topology)

29. Wood, Z.J., Hoppe, H., Desbrun, M., Schröder, P.: Removing excess topology from isosurfaces. ACM Trans. Graph. 23(2), 190-208 (2004)

30. Zomorodian, A., Carlsson, G.: Localized homology. In: Proceedings of the 2007 International Conference on Shape Modeling and Applications, pp. 189-198 (2007) 